\title{
Collaboration Analytics - Current State and Potential Futures
}

\author{
Bertrand Schneider ${ }^{1}$, Nia Dowell2, Kate Thompson ${ }^{3}$
}

\begin{abstract}
This special issue brings together a rich collection of papers in collaboration analytics. With topics including theory building, data collection, modelling, designing frameworks, and building machine learning models, this issue represents some of the most active areas of research in the field. In this editorial, we summarize the papers; discuss the nature of collaboration analytics based on this body of work; describe the associated opportunities, challenges, and risks; and depict potential futures for the field. We conclude by discussing the implications of this special issue for collaboration analytics.
\end{abstract}

\section{Keywords}

Learning analytics, collaborative learning, adaptive systems, data modelling, privacy and ethics, theory

Submitted: 28/02/21 - Accepted: 01/03/21 — Published: 09/04/21

Corresponding author ${ }^{1}$ Email: bertrand schneider@gse.harvard.edu Address: Harvard University, Graduate School of Education, 13 Appian Way, Longfellow Hall 333, Cambridge, MA 02138, USA. ORCID ID: https://orcid.org/0000-0003-0922-2593

${ }^{2}$ Email: dowelln@uci.edu Address: University of California, Irvine, EDUC 3361A, Irvine, CA 92697, USA. ORCID ID: https://orcid.org/00000002-9839-8947

${ }^{3}$ Email: kate.j.thompson@qut.edu.au Address: The University of Melbourne, Melbourne, Victoria, Australia. ORCID ID: https://orcid.org/0000$\underline{0003-0738-0205}$

\section{Introduction}

Collaboration has taken a principal role in educational policy, research, and technology (Graesser et al., 2018), and teams have been recognized as the preferred model for successful economic competitiveness, quality of life, and national security (Fiore, Graesser, \& Greiff, 2018). Research on collaborative learning includes both learning while collaborating and learning to collaborate. The papers in this special issue illustrate the complexity of the area. One purpose of our editorial is to converge on a common understanding of collaboration analytics in order to discuss the potential of its role in research and practice.

A significant barrier to the development of collaboration analytics has been the accessibility of education data in the context of collaboration. Recent developments in data collection techniques now allow researchers and practitioners to collect rich process data on small groups - from clickstreams to multimodal sensor data (Blikstein \& Worsley, 2016), including eyetracking, natural language processing (NLP), motion sensing, and physiological data. The availability of naturally occurring educational data within existing and emerging collaborative environments presents a unique opportunity to make significant advances in our understanding of learners' social, cognitive, and affective interaction dynamics and collaborative ecologies. This opens new doors to both better understand collaborative mechanisms and support them in a rigorous and data-driven fashion (Wise \& Schwarz, 2017). With access to data and developments in artificial intelligence (AI) and machine learning, it is time to pause and consider the potential of collaboration analytics. The opportunities we are most excited by are at the core of this special issue: how can fine-grained data from small groups be leveraged to (1) design better models of collaboration, (2) support interactions between students, (3) design innovative ways of teaching collaborative skills, and (4) contribute to theory building? These new opportunities could radically change research on small groups in education and in the social sciences more generally.

This special issue brings together a rich collection of papers in collaboration analytics. With topics including theory building, data collection, modelling, designing frameworks, and building machine learning models, this issue represents some of the most active areas of research in the field. In this editorial, we summarize the papers; discuss the nature of collaboration analytics based on this body of work; describe associated opportunities, challenges, and risks; and depict potential futures for the field. We conclude by discussing the implications of this special issue for collaboration analytics.

\section{Summary of Papers}

In the first article, Han, Krieger, and Greiff (2021) discuss the importance of definitions and theory for collaboration analytics. 
They point out that (a) current approaches to collaboration analytics fail to take into account and fully capture the comprehensive educational contexts in which students' collaboration takes place and (b) existing definitions and perspectives on collaboration analytics are incongruent. To address this, the authors provide an illustrative example within the context of a collaborative classroom that highlights the co-emergence of three interactions among students, teachers, and content interwoven with time. Their comprehensive framework, which includes the temporal aspects of interaction, learning content, and interactions between students and teachers and among students, offers much potential.

In the second article, Worsley, Anderson, Melo, and Jang (2021) talk about the importance of developing new data collection tools for supporting collaboration analytics. They present a paper describing the development of a tool called BLINC (Building Literacy in In-Person Collaboration) that was informed by data collected from university students regarding their concerns about collaboration. The survey results showed that students' concerns about collaboration were related to seven dimensions that were then used to inform the design of BLINC. This tool is a system of wireless audio/video data collection devices; a centralized server; and a secure, web-based interface for accessing data in real time or post hoc. BLINC is intended to be used not to assess collaboration but instead to frame the conversation for users, giving the group language and permission for discussion. The authors suggest that this process could be used for each new group of end-users to determine the best way to adapt it for different needs. They imagine a future in which teams using a tool such as BLINC are supported to guide their own collaboration in education contexts as well as others.

In the third article, Emara, Hutchins, Grover, Snyder, and Biswas (2021) present a framework for analyzing rich collaboration data. They suggest that collaboration analytics can provide a better understanding of socially shared regulation (SSR) processes by capturing collaborative dialogue, $\log$ files, and task scores. They describe a systematic analysis framework to facilitate the integration of different multimodal data sources. This framework is used in a K-12 setting, where 14 grade 9 students worked in a block-based environment to learn physics by programming specific concepts (e.g., velocity, acceleration). Through NLP, process mining, and a case study, they found that engaging in SSR was associated with more productive coconstruction of knowledge, leading to higher performance scores. The authors argue that their findings contribute to a better understanding of collaborative processes, which can then be used to inform the design of future environments - especially in terms of their ability to provide adaptive feedback during collaborative problem-solving (CPS).

In the fourth article, Swiecki (2021) shifts attention to the importance of interdependence in CPS and associated analytics. The authors point out that a defining feature of CPS is the interdependence that exists between individuals when they work together, that is, how they respond to and influence one another over time. However, few models have been developed that can account for these relationships. These interdependent models seek not to isolate individuals from their team but rather to describe how individuals think and act in relation to each other (e.g., Dowell, Nixon, \& Graesser, 2019; Halpin \& von Davier, 2017). While interdependent models have been found to differ significantly from independent models, that is, those that treat the process of individuals as isolated events by not accounting for how they relate to prior team behaviour, they tend to be more complex. This complexity makes them potentially difficult to use in assessments and systems that need to be explainable for educators, learners, and other researchers. This raises an important issue regarding the trade-off between model complexity and validity, and when researchers and practitioners should deem interdependent models as necessary, or whether simpler models will suffice. To address this, Swiecki (2021) describes the development and testing of a novel quantitative measure of the impact of interdependence that can be used to guide modelling decisions and address specific research questions about collaborative contexts.

In the fifth article, Halpin (2021) focuses attention on dynamical interdependence between members of small groups using data from online chat between dyads collaborating to complete a grade 12 mathematics assessment. Halpin (2021) drew on data generated by automated coding of the chats as containing responses to a question, mathematics content, or chat messages with no mathematics content. A Markov chain was used to model group dynamics, which were then related to group outcomes, while pre-test scores and word count were used as the control variables. The paper aims to provide a simple model with indepth discussion of reliability and validity for other researchers to apply to more complex models of learning and collaboration.

In the sixth article, Lämsä et al. (2021) use cutting-edge supervised machine learning models to automatically predict phases of collaboration. They collected data during a computer-supported collaborative inquiry-based learning (CSCIL) activity, where 55 students learned about thermodynamics in groups of five. By manually coding students' interactions, they labelled the data according to the five phases of an inquiry-based learning (IBL) cycle (orientation, conceptualization, investigation, conclusion, and discussion). They then used transcript data to train deep network models. These models are stateof-the-art supervised machine learning models that perform well on a variety of prediction tasks. They found that their best model was slightly better than the human coder $(58.9 \%$ vs. $54.3 \%)$. They conclude by describing the potential of this approach for real-time support of CSCIL activities, by combining their models with speech recognition technologies.

In the seventh and final article, Martinez-Maldonado and colleagues (2021) highlight the need to use human-centred designs when designing collaboration analytics. They draw on substantial literature and their previous research to provide a conceptual model that they then apply to four cases. The authors envision a future in which researchers theorize how 
collaboration constructs relate to activity traces and how the designers of collaboration analytics tools would produce useful tools for educators and students. Each of the cases, including educational settings such as high school, undergraduate university, and graduate school, addressed the core elements of collaboration analytics included in the proposed conceptual framework: theory, task design, human-centred design, and the generation of actionable group insights. A substantial contribution of this paper is the attention the authors give to what they call the opportunities and challenges but are essentially key considerations when making design decisions about collaboration analytics.

\section{What Is Collaboration Analytics?}

Collaborative learning has been broadly defined as "a situation in which two or more people learn or attempt to learn something together" (Dillenbourg, 1999). Learning analytics is defined as "the measurement, collection, analysis, and reporting of data about learners and their contexts, for the purposes of understanding and optimizing learning and the environments in which it occurs" (Siemens, 2013). Thus, we can consider that collaboration analytics (applied to educational settings) is the intersection of these two definitions: it expands the definition of learning analytics to focus on groups of students, or adds the idea of measuring, collecting, and analyzing data from groups of learners for the purpose of supporting them. This echoes the definition of computer-supported collaborative learning (CSCL), which has been defined as collaborative learning in which technology plays a substantial role (Goodyear, Jones, \& Thompson, 2014), but puts data analytics at the forefront. Each paper in the special issue, however, defines collaboration analytics slightly differently, depending on the authors' particular epistemological stance about learning, collaboration, and learning analytics. Such differences can lead to nuanced disagreements and are part of the tension when people from different fields come together to create something new. Learning analytics researchers are well practised at connecting analytic techniques to deep theoretical perspectives (Wise \& Schwarz, 2017), and the richness of these epistemological stances has been generating innovative and interesting work, as exemplified by the papers in this special issue.

All the authors involved in this special issue, ourselves included, have their own approach to defining collaboration analytics. We draw on these papers, as well as other literature, to identify eight areas of active research in collaboration analytics. As highlighted in Figure 1, we suggest that collaboration analytics must draw on theories of collaboration and inform the creation of new data collection tools to build computational models of interactions, leading to new understandings of collaboration and informing the development of predictive models for collaborative states. Collaboration analytics can then inform the design of support systems, including stakeholders in the design process, and ensure the specific considerations of privacy and ethics. We review each of these areas below.

As can be seen in Figure 1, collaboration analytics can contribute to both research and practice. In considering the relationship between collaboration analytics and research, the first focus of collaboration analytics discussed here is to leverage and contribute to theories of collaboration. We won't discuss theories related to collaboration and collaborative learning, because as both Han and colleagues (2021) and Martinez-Maldonado and colleagues (2021) found, the existing models tend to be incomplete and complex. Interdisciplinary collaboration is an area in which multiple theories have been aligned to provide a model of collaborative learning (e.g., Pennington et al., 2016; Markauskaite et al., 2020). In these studies, collaborative learning involves interpreting, responding to, and working with others; interdisciplinary collaboration is viewed as an evolving practice. To inform the refinement of theoretical models of interdisciplinary collaboration, we need to collect multiple types of data to observe what learners are saying and doing as well as apply analytic techniques to identify productive collaboration (e.g., Gosselin, Thompson, Pennington, \& Vincent, 2020; Pennington, Vincent, Gosselin, \& Thompson, in press). The application of collaboration analytics can inform the development of theory about collaborative learning in many different learning situations.

A second focus is on the development of innovative data collection tools that provide new ways of capturing, understanding, and supporting social interactions. Over the last decade, the availability of larger and richer datasets has transformed many fields of research and, arguably, our society as a whole. From genetic sequencing to personal recommendation systems, big data is changing the way we look at the past to understand the present and predict the future. Collaboration analytics is a perfect example of this - by developing new data collection tools that allow us to visualize social interactions in real time, it could allow students to better understand group interactions and how to interact with others. The BLINC platform (Worsley et al., 2021) is a first step in this direction. By providing information about the group's discussion, the tool allows students to start a conversation on how their collaborative processes could be improved. The authors ground their design in a user-centric approach, where survey questions captured students' concerns when collaborating in small groups. Looking beyond this special issue, the development of new data collection tools is an exciting and fast-moving subfield of collaboration analytics. While the cost of sensing devices used to be prohibitive, the last 20 years has seen more and more inexpensive hardware be commercialized. For example, one can now purchase an eye-tracker for a few hundred dollars, which is an order of magnitude less than decades ago; fitness trackers like the Fitbit or motion sensors like the Kinect Azure are in a similar price range. We are also seeing integration of those sensors into everyday devices, with newer versions of the iPhone 
or Microsoft Hololens that have built-in eye-tracking capabilities. More recently, better and better computer vision algorithms are starting to replace physical sensing devices entirely. As an example of a toolkit that doesn't require any special hardware to collect multimodal data, the EZ-MMLA website (Hassan, Leong \& Schneider, 2021) allows anyone with a webcam to generate gaze, motion, and physiological data from a browser, which can then be used to collect rich data from educational contexts. This "data deluge" presents new challenges in cleaning, sanity-checking, post-processing, and fusing (Ochoa \& Worsley, 2016) datasets from different sources, but it also creates new opportunities for understanding collaborative interactions.

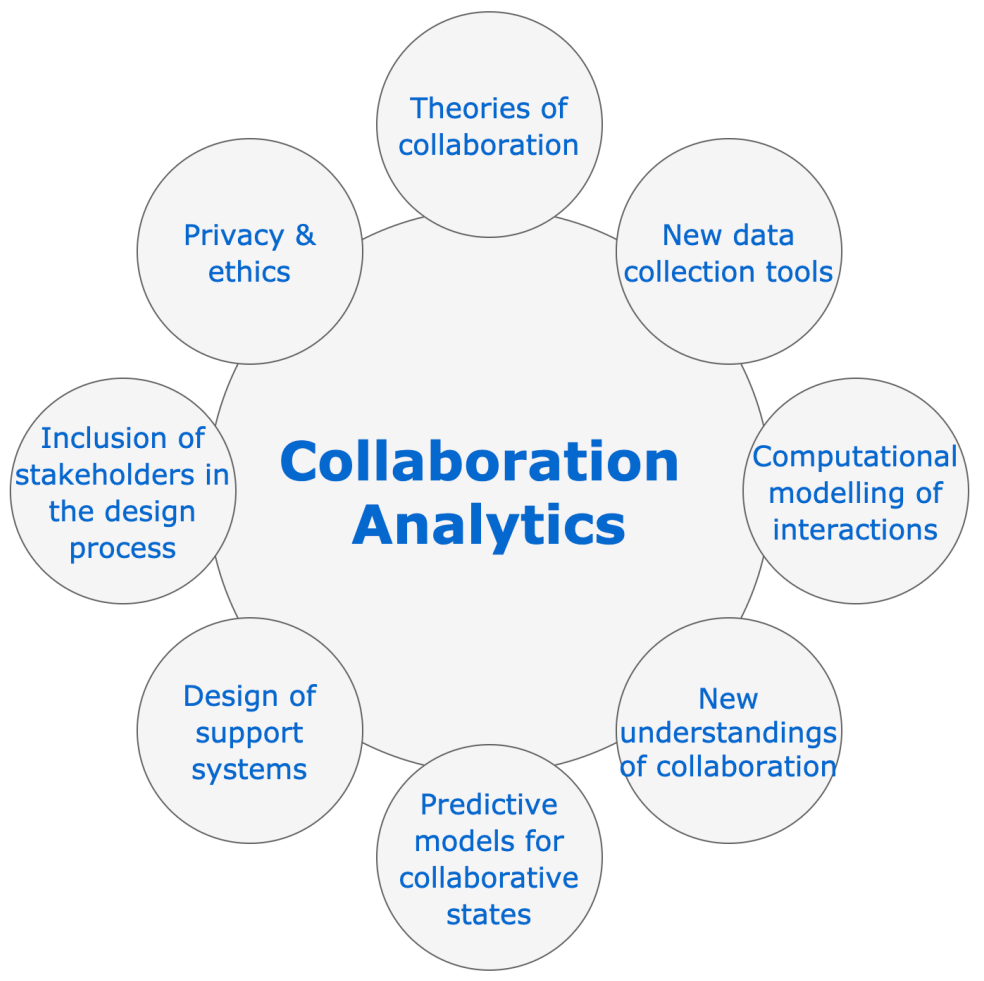

Figure 1. An overview of active areas of research in collaboration analytics.

A third focus is on leveraging these large datasets through computational models. Researchers have explored various approaches, some of them featured in this special issue. Lämsä and colleagues (2021), for example, trained supervised machine learning (i.e., deep neural networks) models to predict different phases of the collaborative process. Supervised machine learning has been successfully used for a variety of tasks in many domains - however, the majority of these predictions tend to be low-level, observable events easily coded through crowd-sourcing services (e.g., tagging objects or people). A challenge of collaboration analytics is to predict social constructs that are not easily identifiable and require coding schemes with high validity and reliability, which increases the cost of generating large datasets with labelled instances. These challenges are visible in Lämsä and colleagues' (2021) work, where human coders struggled to perfectly identify phases of a CSCIL activity. Nevertheless, there is promise in leveraging supervised models for predicting collaborative states, especially as we develop better and more efficient ways of labelling data.

A fourth focus is on understanding collaborative processes through fine-grained data and computational models. In collaboration analytics, the primary phenomenon of interest may span many grain sizes. In this context, it has been widely acknowledged that collaboration and collaborative processes reside at varying levels and take place across different phases of collaboration. That is, collaborative interactions can be analyzed at the level of the group or the individual student, between students, and at the cultural level (Janssen, Erkens, \& Kirschner, 2011). Emara and colleagues (2021) explored this issue in the context of students' SSR processes. Their systematic analysis framework highlights the importance of capturing multimodal representations of regulatory processes that take place on varying levels, namely on an individual (self-regulation) and on a group (SSR) level (Hadwin, Järvelä, \& Miller, 2011; Wise \& Shaffer, 2015). Halpin (2021) investigates models of dynamical interdependence in dyads, accounting for both individual and group measures in the model. Swiecki (2021) takes a different lens and explores the potential importance of interdependence in collaboration analytics, that is, how individuals respond to and influence one another over time during collaborative interactions. Their work was situated within the definition of collaboration analytics provided by Martinez-Maldonado and colleagues (2019), wherein collaboration analytics refers to the 
particular techniques and approaches needed to automatically (or semi-automatically) capture, analyze, mine, and distill data about collaborators' interactions. The authors suggest that many current models of collaboration obfuscate any details about the moment-to-moment interactions between the individual and their team, how individuals may depend on one another for information, and how they may influence one another over time. To address this, Swiecki (2021) describes the development and testing of a novel quantitative measure of the impact of interdependence that can be used to guide modelling decisions and address specific research questions about collaborative contexts. The results suggest that the measure can distinguish between individuals who have been impacted by interdependence differently, that interdependence is impactful in their data overall, and that aspects of the task design may have affected the impact of interdependence on some individuals.

A fifth focus is the inclusion of key stakeholders in the design of analytics. Martinez-Maldonado and colleagues (2021) discuss this challenge, especially the end users of collaboration analytics in the design process, and Worsley and colleagues (2021) discussed the development of a tool designed to be altered in response to the varying needs of the users. Collaboration between researchers, developers, teachers, and learners is a key element of methodologies common in the learning sciences, such as design-based research (Brown, 1992; Reimann, 2013) and research-practice partnerships (Penuel \& Gallagher, 2017). Both are related to investigating problems of practice and are intended to support a collaborative approach between practitioners and researchers. In order for collaboration analytics to realize the potential discussed in many of the papers in this issue of generating new theory, inclusion of key stakeholders is vital in the development of tools and their implementation in authentic learning situations.

Collecting additional types of data in new contexts to explore social interactions between students, however, brings new challenges. Data privacy and ethics have become a central focus in the learning analytics community (and beyond), particularly when the data is shared between students within a group, but also because the data collected is from students in learning situations which can be challenging and frustrating and involves students in vulnerable situations. The quality of the data is also a major concern; the easy availability of "cheap" data can influence researchers to focus on less relevant constructs (without leveraging existing learning theories; see concerns raised by Wise \& Shaffer (2015)), and common standards for collecting and reporting on learning data do not currently exist. Finally, new approaches also come with new biases (O'Neil, 2016), from black box models that are fine-tuned for specific samples of students and underperform for others, to researchers' attention being drawn to incomplete or misleading information (such as the streetlight effect; Ochoa \& Worsley (2016)).

In summary, the different foci of this special issue (Figure 1) represent areas of research where collaboration analytics can make significant contributions. In the next section, we discuss opportunities and challenges for these contributions.

\section{Opportunities and Challenges for the Field of Collaboration Analytics}

Collaboration analytics can impact the future of collaborative learning and collaborative work, in terms of both research and practice. In this section, we describe the potential benefits (and dangers) of using a data-driven, analytical approach.

Many technical challenges to the implementation of collaboration analytics have been addressed, but some aspects of the way they are used in the practice of research about collaboration still impact their current utility. In order for collaboration analytics to help researchers better understand collaboration, we suggest that the practice needs to converge in five key areas. Agreed-upon, common definitions of collaboration analytics and collaboration are lacking. Seven articles are included in this special issue, with differing perspectives even on a holistic view of what is important in collaboration analytics. Han and colleagues (2021) consider this to include teacher, student, time, etc.; Martinez-Maldonado and colleagues (2021) include a variety of elements from a design framework; and Swiecki (2021) and Halpin (2021) both identify the importance of interdependence. Worsley and colleagues (2021) and Emara and colleagues (2021) discuss multimodal collaboration analytics - focusing on measures of learning without reference to other elements in the learning situation. Without a shared understanding of the phenomenon studied, it is challenging to relate findings and advance the field. Common ways of generating datasets that can be shared between researchers could provide a basis for the creation of a shared understanding of collaboration. Tools could then be created to build consistency and provide researchers with an approach to the implementation of this data-driven approach.

With some reservations about potential confusion, this suggests that a collaborative approach to research about collaboration could be adopted. The research practices in interdisciplinary fields such as environmental science have evolved over the last 10 years with a recent focus on convergence in science. Pennington and colleagues (2016) show that key stages include (1) identification of an appropriate research question, (2) agreement on a shared vocabulary, (3) the co-creation of boundary negotiating objects, and (4) tools for visualizing and combining data, with (5) the aim of producing a new, connected model of understanding. If this model is adopted, then in developing and implementing collaboration analytics, researchers, practitioners, and learners will need to collaborate in order to agree on our common questions using a shared vocabulary, cocreating the tools we need to visualize and combine data. A collaborative approach to research in collaboration, supported by collaboration analytics, means that we are better placed to make contributions to both theory and practice. We imagine this as 
an ongoing, evolving conceptualization of collaboration — beginning with a shared model of collaboration that is tested in various learning situations supported by collaboration analytics, which are then used to support the revision of the model, in which collaboration analytics tools, etc., are revised and reapplied. This approach would mean that the models of collaboration that researchers use to inform their inquiry would be articulated and shared with partners, standard tools would be used to collect the data, and researchers would collaborate to ensure representative samples are accessible and utilized. Creation of shared visualizations would also help researchers understand how different data, the collection of which was informed by different epistemological perspectives, can be connected to produce a new model of understanding of the phenomena.

From a practical perspective, a defining component of collaboration analytics is to support collaboration using data in ecological settings. Collaboration analytics is about going beyond describing and understanding collaborative processes to design systems that can help key stakeholders. In education, the Journal of Learning Analytics community is primarily interested in supporting individual learners, small groups of students, communities, and teachers. All of the authors of this special issue emphasize the importance of collaboration analytics for practice. Emara and colleagues (2021) suggest that their work can "inform the design of future environments and tasks to foster better collaboration and learning in computational scientific modelling and beyond [and that] [T] here is increasing interest in designing adaptive support and timely feedback for CPS." Along the same line, Lämsä and colleagues (2021) mention that their "methodology may be combined with automatic speech recognition methods to analyze face-to-face conversations and support real-time orchestration of CSCIL activities," noting that their findings "may advance the design of adaptive guidance of CSCIL; visualizations of the results could be presented in the dashboards of teachers or students [...] From the teachers' perspective, these dashboards could support their orchestration of CSCIL activities in real time (e.g., facilitating the guidance of students and provision of feedback) [...] From the students' perspective, these dashboards could be used as awareness tools." This is echoed by Worsley and colleagues (2021), who envision teams using a tool such as BLINC to "guide collaboration." Building on this, Martinez-Maldonado and colleagues (2021) ponder how collaboration analytics designers could produce software tools that make sense to educators and students. Those considerations are further reflected upon by Han and colleagues (2021), who argue for an ecological approach, where collaboration analytics is holistic and contextually sensitive. In summary, the contributors to this special issue are all optimistic about the use of collaboration analytics in naturalistic settings.

An ideal future of collaboration analytics is to develop systems that are theory based, collect rich interaction data, model collaboration in adaptive ways, are context sensitive, respect users in ethical ways, and support users at different levels based on individual and group characteristics. The field needs to conceptualize different levels of support and get a better understanding of how they should be applied. A useful starting point is the framework by Soller, Martínez, Jermann, and Muehlenbrock (2005). They describe a four-level model where increasingly complex support is provided to groups of learners. In the first level, interaction data is collected and aggregated to offer new insights on collaborative processes. In the second level, the current state of interaction is mirrored to the group; the group then decides whether their state is productive or needs to be regulated. These mirroring tools have been successfully used to promote awareness and reflection in small groups, for example, in terms of equity of verbal participation (Bachour, Kaplan \& Dillenbourg, 2010) or as dashboards for teachers (Schwendimann et al., 2016). The third level compares the current state of interaction to a "desired state" and offers metacognitive tools to learners (e.g, by displaying information about what the desired interaction state might look like along with a visualization of their current state). Finally, in the fourth level, the system guides the group toward more productive states. This kind of system needs to know not only what a desired state looks like but also how to get there. An example of a guiding system that makes a first step in this direction is the EPCAL platform (Hao et al., 2017), where an intelligent facilitator provides high-level prompts for group members to stay on topic and be polite, engaged, and responsive to others when they are perceived to be lacking in these areas.

A potential future for collaboration analytics is to build models that can work on those different levels and can provide adaptive support based on the needs and skills of the group. This potential can be fulfilled by doing some groundwork in using common theories and definitions, designing new ways of operationalizing collaborative processes, developing new data collection tools, modelling social interactions in more sophisticated ways, designing interfaces that are user centred and context sensitive, and building smart systems that can adapt to various group dynamics. These opportunities are where the field of collaboration analytics can make significant contributions.

\section{Dangers and Challenges with Pushing These Approaches Too Far}

Although the authors in this special issue present several promising avenues for collaboration analytics, the work poses some technical and ethical considerations. For instance, Han and colleagues (2021) present a more utopian view of collaboration analytics that is holistic and contextually sensitive. Their comprehensive framework, which includes the temporal aspects of interaction, learning content, and interactions between students and teachers and among students, offers much potential. One can imagine a number of possibilities coming to life when combining this holistic lens with recent technological advances, 
such as body movement sensing, speech to text, and facial recognition. For instance, smart collaborative activities in classrooms equipped with such technologies could provide both teachers and students the ability to monitor their behavioural engagement (listening, speaking, or disengaged) and their social, cognitive, and affective processes, and simultaneously measure and compare teachers' actions and students' performance or levels of collaboration. However, some technical challenges must be solved, such as difficulties incorporating mass data from different sources in a synchronized way, not overwhelming teachers and students with too much data that is ineffectively communicated, and appropriately making latent variables visible (e.g., "mental regulation processes"). It might only be possible for research to adopt such considerations with respect to the analysis of small groups or a small number of course units due to time and resource limitations.

Additionally, several ethical considerations come to mind when navigating the implementation of comprehensive tracking of students in classrooms, as well as the collaboration analytics topics across the articles in this special issue, more broadly. These issues primarily relate to data collection (e.g., privacy, ethics, consent), modelling (e.g., inclusion, accuracy), algorithms (e.g., bias, accountability) (ACM US Public Policy Council, 2017), and data management (e.g., data ownership, provenance, storage), none of which are likely to be unique to collaboration analytics, but instead have ethical dimensions of analytics that require deliberate consideration and proactive planning by the broader learning analytics community (Drachsler et al., 2015; Wise, 2019).

Some of these issues, which are present in the wider learning analytics and collaboration analytics research, are evident in the special issue articles, namely predictive modelling and the use of NLP (e.g., Lämsä et al., 2021). For instance, Lämsä and colleagues (2021) provide a novel exploration of face-to-face collaborative inquiry-based learning interactions. Their approach utilized human content analysis of discourse, cutting-edge NLP, and supervised machine learning models (i.e., word embeddings and deep neural networks (DNN) with attention layers) to automatically predict phases of collaboration. Their study could advance the design of adaptive guidance of CSCIL, wherein visualizations of the results could be presented in teachers' or students' dashboards. Despite the potential for this and similar efforts within the field, we as a community should be aware of and ideally address several non-trivial issues before widespread adoption can be realized.

These recent advancements in artificial neural networks (ANNs) and deep learning have resulted in the development of these highly effective techniques for discourse representation and collaboration analytics. A major player in the field was revealed by Google and is a breakthrough AI technology called BERT (Bidirectional Encoder Representations from Transformers; Devlin, Chang, Lee, \& Toutanova, 2019), which has garnered significant attention in the machine learning community by presenting state-of-the-art results in a wide variety of NLP tasks, including question answering, natural language inference, and others. BERT's main technical innovation is applying the bidirectional training of Transformer, a widely used attention model, to language modelling. This is in contrast to previous efforts, which examine a text sequence either from left to right or combined for training. Devlin and colleagues (2019) highlight how a bidirectionally trained language model can have a deeper sense of language context and flow than single-direction language models. However, this revolutionary AI appears to have a significant issue, as articulated by The New York Times: "It could be picking up on biases in the way a child mimics the bad behavior of his parents" (Metz, 2019). That is, BERT, like many other similar NLP approaches, learns linguistic representations from tons of digitized information, such as old books, Wikipedia entries, and news articles. This has created non-trivial issues since these societal artifacts carry decades of biases as well as the current biases within our society (Callahan \& Herring, 2011). An illustration of the problematic behaviour is the recurrently appearing occupational stereotypes that the word homemaker is related to the word woman as the word programmer is to the word man (Bolukbasi, Chang, Zou, Saligram, \& Kalai, 2016; Webster, Costa-jussà, Hardmeier, \& Radfor, 2019). Recent studies have aimed to detect, analyze, and mitigate gender bias in different NLP tools and applications including word embeddings, but these issues remain and should be carefully thought about when implementing any NLP techniques.

Similar issues can be seen with the application of predictive models in the context of collaboration analytics. That is, all learning analytics are based on models that are an approximation to the actual learning activity (Kitto, Buckingham Shum, \& Gibson, 2018), and collaboration/teamwork introduces more modelling complexity than individual learning tasks. Winne (2017, p. 8) has warned the community about the concerns and challenges in attempting to help learners using predictive models based on incomplete data. He suggests it can be "quite hazardous to predict what will happen to a particular learner" based on big data. We argue that for the case of collaboration analytics, it would be even more risky trying to find causal relationships in incomplete data about such a complex phenomenon. Yet, even if we are able to create better predictive models based on more data traces, this would also increase the complexity of (i) the underlying collaboration models and (ii) the complexity of user interfaces for educators and students (Martinez-Maldonado, Schulte, Echeverria, Gopalan, \& Buckingham Shum, 2020).

\section{What Are the Potential Futures of Collaboration Analytics?}

While there are exciting opportunities for research and practice, we also wonder about the future of the field when we integrate 
those different research strands. Looking across the papers of this special issue, all the authors discuss the future of the field in terms of informing the design of smart adaptive systems that could coach groups and teach collaboration skills. While this is a possibility, we want to take the time to examine what their proposed future would look like and consider alternatives. To do this, we adopt the framing of intelligent tutoring systems (ITSs). ITSs have been successful at teaching difficult concepts in closed-end domains (i.e., where there is an objective right or wrong answer to a problem). These systems have been making considerable gains in accurately tracking students' knowledge and offering guidance to address misconceptions. In the previous section, we described a framework (Soller et al., 2005) that breaks down the steps to build this kind of smart system. In Table 1, we expand this model by describing a continuum between unintelligent and smart adaptive systems for supportive collaboration. The first row describes the features necessary for these levels of intelligence (e.g., capturing and displaying collaborative states; inferring, predicting, and adjusting group support). The second row mentions benefits of each level, such as getting access to richer interaction data, supporting group reflection, diagnosing the group's state, and providing advice. The third and fourth rows reflect on the opportunities and challenges associated with each level, especially in terms of developing diagnostic models of collaboration. The final two rows describe the main area of contribution and examples of systems that have implemented some of these features.

Table 1. A possible future for the field of collaboration analytics. The first row describes a continuum from unintelligent to smart adaptive systems, with associated features, opportunities, challenges, and examples.

\begin{tabular}{|c|c|c|c|c|c|}
\hline Continuum & $\begin{array}{l}\text { Unintelligent } \\
\text { system }\end{array}$ & $\begin{array}{l}\text { Mirroring } \\
\text { system }\end{array}$ & $\begin{array}{l}\text { System with } \\
\text { meta-cognitive } \\
\text { tools }\end{array}$ & $\begin{array}{l}\text { Guiding } \\
\text { system }\end{array}$ & $\begin{array}{l}\text { Smart adaptive } \\
\text { system }\end{array}$ \\
\hline Features & $\begin{array}{l}\text { captures current } \\
\text { state }\end{array}$ & $\begin{array}{l}\text { displays current } \\
\text { state }\end{array}$ & $\begin{array}{l}\text { infers desired } \\
\text { state }\end{array}$ & $\begin{array}{l}\text { predicts next } \\
\text { best action to } \\
\text { reach desired } \\
\text { state }\end{array}$ & $\begin{array}{l}\text { adjusts guidance } \\
\text { based on contexts } \\
\text { and users }\end{array}$ \\
\hline Allows for & $\begin{array}{l}\text { analyzing rich } \\
\text { interaction data }\end{array}$ & $\begin{array}{l}\text { monitoring and } \\
\text { reflecting on } \\
\text { group } \\
\text { interactions }\end{array}$ & $\begin{array}{l}\text { comparing } \\
\text { "current" with } \\
\text { "ideal" state }\end{array}$ & $\begin{array}{l}\text { providing } \\
\text { advice on how } \\
\text { to work } \\
\text { together }\end{array}$ & $\begin{array}{l}\text { adapting to vastly } \\
\text { different group } \\
\text { dynamics and } \\
\text { domains }\end{array}$ \\
\hline Opportunities & $\begin{array}{l}\text { develop new } \\
\text { data collection } \\
\text { tools }\end{array}$ & $\begin{array}{l}\text { develop user- } \\
\text { centred } \\
\text { visualizations } \\
\text { and designs to } \\
\text { support small } \\
\text { groups }\end{array}$ & $\begin{array}{l}\text { develop } \\
\text { diagnostic } \\
\text { models of } \\
\text { collaboration }\end{array}$ & $\begin{array}{l}\text { develop } \\
\text { predictive } \\
\text { models to } \\
\text { guide groups } \\
\text { toward a } \\
\text { better state }\end{array}$ & $\begin{array}{l}\text { develop holistic } \\
\text { and context-aware } \\
\text { systems }\end{array}$ \\
\hline Challenges & $\begin{array}{l}\text { collect high- } \\
\text { quality (e.g., } \\
\text { beyond } \\
\text { clickstream) } \\
\text { data while } \\
\text { addressing } \\
\text { issues of ethics } \\
\text { and privacy }\end{array}$ & $\begin{array}{l}\text { minimize } \\
\text { cognitive load } \\
\text { while providing } \\
\text { useful insights } \\
\text { about } \\
\text { collaborative } \\
\text { interactions }\end{array}$ & $\begin{array}{l}\text { identify "ideal" } \\
\text { states by taking } \\
\text { individual and } \\
\text { group } \\
\text { differences into } \\
\text { account }\end{array}$ & $\begin{array}{l}\text { identify next } \\
\text { best action(s) } \\
\text { to get closer } \\
\text { to an "ideal" } \\
\text { state }\end{array}$ & $\begin{array}{l}\text { develop models } \\
\text { that are context } \\
\text { independent (i.e., } \\
\text { can generalize to } \\
\text { any situation) }\end{array}$ \\
\hline Contributions & data collection & $\begin{array}{l}\text { data } \\
\text { visualization }\end{array}$ & data modelling & AI & AI \\
\hline Examples & $\begin{array}{l}\text { EZ-MMLA } \\
\text { (Hassan et al., } \\
\text { 2021) }\end{array}$ & $\begin{array}{l}\text { BLINC } \\
\text { (Worsley et al., } \\
2021 \text { ) }\end{array}$ & $\begin{array}{l}\text { Some } \\
\text { dashboards } \\
\text { (Schwendimann } \\
\text { at al., 2016) }\end{array}$ & $\begin{array}{l}\text { EPCAL (Hao } \\
\text { at al., 2017) }\end{array}$ & None \\
\hline
\end{tabular}


While this approach to framing the steps involved in the construction of a smart system has been successful for ITS research in closed-end domains, a reasonable question is whether this is the only (or even the best) way forward for collaboration analytics. Unintelligent systems and mirroring systems are relatively easy to design (compared to smarter systems), but they face important issues of privacy and ethics. However, in collaboration analytics, the levels that include meta-cognitive or guiding capabilities are creating important bottlenecks. Because there is no right or wrong answer for collaborative interactions (beyond detecting obvious non-productive behaviours, such as the free rider or sucker effects; Salomon \& Globerson (1989)), it is difficult to develop sophisticated guiding systems. At best, some of these systems will work only in a specific context, without easily generalizing to other settings. Given the difficulty of the task, a question is whether the field needs to consider alternative futures that add to the model that the ITS research has used.

Two options that we considered draw on an understanding of collaborative learning that acknowledges the role of the teacher. The first is the ASSISTments platform (described in Baker, 2016), where teachers play an active role in supporting students by looking at their interactions with a simple tutoring system. A similar approach could be used for collaboration, where a facilitator or expert could be an intermediary between the smart system and students. Another example is the Mursion platform (Hudson, Voytecki, \& Zhang, 2018), where teacher candidates are immersed in a virtual world where they have to manage a classroom of students. The students are "stupid" agents in the sense that the AI can only display simple behaviours. However, the differentiating factor is that a human "puppeteer" can control students when necessary to create interesting professional development scenarios (such as a student disrupting the class, lagging behind, or being disengaged). The aspirant teacher then reacts in real time to address the issue and move on with the class. This kind of real-world interaction would be difficult and costly to create in an actual classroom, but the combination of a virtual world with simple AI agents (controlled by an experienced teacher) turns it into a powerful experience. The analytics can be used to support the learner and more experienced teachers, for example, during the session (to suggest interesting scenarios or courses of action) or after the session (to reflect on the choices made and offer guidance on considering alternatives). One can imagine this kind of scenario being transferred to the learning of collaborative skills, which opens new opportunities for thinking about the use of collaboration analytics.

\section{Conclusion}

In this editorial we have summarized the papers of the special issue; discussed the nature of collaboration analytics based on this body of work; described associated opportunities, challenges, and risks; and depicted potential futures for the field. We brought together definitions of collaboration, learning analytics, and CSCL to define collaboration analytics. We also considered the opportunities, needs, and challenges for the field. Finally, we described multiple futures for collaboration analytics, beyond smart adaptive systems. Through these different lenses, we hope that this editorial can generate productive conversations about the current state and future of the field.

The quality of the contributions to this special issue speaks for itself, so there is no need for additional praise. Nevertheless, there is a need to point out that the field (as any field of research) suffers from some blind spots, and that much work remains to be done. There is arguably wide agreement that the field of collaboration analytics is both an important and an interesting one, and its current state suffers from bottlenecks that result in unrealized potential. As scientists - and this special issue is a great example of this current state - have begun to understand the mechanisms and the potential underlying collaboration analytics, the broader applications and larger exploitation are still some distance away. This is by no means meant as a discouragement but rather an encouragement to the field, and some big leaps, shifts in perspective, and steady development are likely to continue throughout the near future. We conclude by leaving the reader with the following scenario, which serves as an illustrative example based on a synthesis of the topics covered in this special issue. Imagine a classroom equipped with human-centred design-based multimodal sensors that provide visualizations of real-time teacher, student, and content interaction analytics, over time, in a manner that is sensitive and revealing of the interdependent relationship between all these interactions. Imagine that as the system learns about the people and how they learn with each other, the students and teacher also learn about themselves. In this imagined future, what new questions would inspire us, as researchers, about the role of collaboration in learning?

\section{Declaration of Conflicting Interest}

The authors declared no potential conflicts of interest with respect to the research, authorship, and/or publication of this article.

\section{Acknowledgements}

The guest editors would like to thank the journal managers and the staff of the Journal of Learning Analytics for their support in all practicalities. The guest editors would also like to thank the authors and reviewers, without whom this special issue would never have come to pass. 


\section{References}

ACM US Public Policy Council. (2017). Statement on algorithmic transparency and accountability. Communications of the $A C M$. https://www.acm.org/binaries/content/assets/public-policy/acm-pres-ltr-un-re-weapons-systems.pdf

Bachour, K., Kaplan, F., \& Dillenbourg, P. (2010). An interactive table for supporting participation balance in face-to-face collaborative learning. IEEE Transactions on Learning Technologies, 3(3), 203-213. https://doi.org/10.1109/TLT.2010.18

Baker, R. S. (2016). Stupid tutoring systems, intelligent humans. International Journal of Artificial Intelligence in Education, 26(2), 600-614. https://doi.org/10.1007/s40593-016-0105-0

Blikstein, P., \& Worsley, M. (2016). Multimodal learning analytics and education data mining: Using computational technologies to measure complex learning tasks. Journal of Learning Analytics, 3(2), 220-238. https://doi.org/10.18608/jla.2016.32.11

Bolukbasi, T., Chang, K.-W., Zou, J., Saligrama, V., \& Kalai, A. (2016). Man is to computer programmer as woman is to homemaker? Debiasing word embeddings. In Proceedings of the 30th International Conference on Neural Information Processing Systems (NIPS 2016), 5-10 December 2016, Barcelona, Spain (pp. 4356-4364). Red Hook, NY, USA: Curran Associates. https://dl.acm.org/doi/10.5555/3157382.3157584

Brown, A. L. (1992). Design experiments: Theoretical and methodological challenges in creating complex interventions in classroom settings. The Journal of the Learning Sciences, 2(2), 141-178. https://doi.org/10.1207/s15327809jls0202_2

Callahan, E. S., \& Herring, S. C. (2011). Cultural bias in Wikipedia content on famous persons. Journal of the American Society for Information Science, 62(10), 1899-1915. https://doi.org/10.1002/asi.21577.

Devlin, J., Chang, M.-W., Lee, K., \& Toutanova, K. (2019). BERT: Pre-training of deep bidirectional transformers for language understanding. In Proceedings of the 2019 Conference of the North American Chapter of the Association for Computational Linguistics: Human Language Technologies, Volume 1 (Long and Short Papers), 2-7 June 2019, Minneapolis, MN, USA (pp. 4171-4186). https://doi.org/10.18653/v1/N19-1423

Dowell, N. M., Nixon, T., \& Graesser, A. C. (2019). Group communication analysis: A computational linguistics approach for detecting sociocognitive roles in multi-party interactions. Behavior Research Methods, 51(3), 1007-1041. https://doi.org/10.3758/s13428-018-1102-z

Dillenbourg, P. (1999) What do you mean by collaborative learning?. In P. Dillenbourg (Ed.), Collaborative Learning. Cognitive and Computational Approaches (pp. 1-19). Oxford, UK: Elsevier.

Drachsler, H., Hoel, T., Scheffel, M., Kismihók, G., Berg, A., Ferguson, R., ... Manderveld, J. (2015). Ethical and privacy issues in the application of learning analytics. In Proceedings of the Fifth International Conference on Learning Analytics and Knowledge (LAK '15), 16-20 March 2015, Poughkeepsie, NY, USA (pp. 390-391). New York: ACM. https://doi.org/10.1145/2723576.2723642

Emara, M., Hutchins, N. M., Grover, S., Snyder, C., \& Biswas, G. (2021). Examining student regulation of collaborative, computational, problem-solving processes in open-ended learning environments. Journal of Learning Analytics, 8(1), 49-74. http://doi.org/10.18608/jla.2021.7230

Fiore, S. M., Graesser, A., \& Greiff, S. (2018). Collaborative problem-solving education for the twenty-first-century workforce. Nature Human Behaviour, 2(6), 367-369. https://doi.org/10.1038/s41562-018-0363-y

Goodyear, P., Jones, C., \& Thompson, K. (2014). Computer-supported collaborative learning: Instructional approaches, group processes and educational designs. In J. Spector, M. Merrill, J. Elen, \& M. Bishop (Eds.), Handbook of Research on Educational Communications and Technology (pp. 439-451). New York: Springer. https://doi.org/10.1007/978-1-4614-3185-5 35

Gosselin, D. C., Thompson, K., Pennington, D., \& Vincent, S. (2020). Learning to be an interdisciplinary researcher: Incorporating training about dispositional and epistemological differences into graduate student environmental science teams. Journal of Environmental Studies and Sciences, 10, 310-326. https://doi.org/10.1007/s13412-020-00605-W

Graesser, A. C., Fiore, S. M., Greiff, S., Andrews-Todd, J., Foltz, P. W., \& Hesse, F. W. (2018). Advancing the science of collaborative problem solving. Psychological Science in the Public Interest: A Journal of the American Psychological Society, 19(2), 59-92. https://doi.org/10.1177/1529100618808244

Hadwin, A. F., Järvelä, S., and Miller, M. (2011). Self-regulated, co-regulated, and socially shared regulation of learning. In B. J. Zimmerman \& D. H. Schunk (Eds.), Handbook of Self-Regulation of Learning and Performance (pp. 65-84). New York: Routledge. https://psycnet.apa.org/record/2011-12365-005

Halpin, P. (2021). Measuring dynamical interdependence in small-group collaborations. Journal of Learning Analytics, 8(1), 95-112. http://doi.org/10.18608/jla.2021.7241

Halpin, P. F., \& von Davier, A. A. (2017). Modeling collaboration using point processes. In A. A. von Davier, M. Zhu, \& P. C. Kyllonen (Eds.), Innovative Assessment of Collaboration (pp. 233-247). Springer. https://doi.org/10.1007/978-3319-33261-1 15

ISSN 1929-7750 (online). The Journal of Learning Analytics works under a Creative Commons License, Attribution - NonCommercial-NoDerivs 3.0 Unported (CC BY-NC-ND 3.0) 
Han, A., Krieger, F., \& Greiff, S. (2021). Collaboration analytics need more comprehensive models and methods. An opinion paper. Journal of Learning Analytics, 8(1), 13-29. http://doi.org/10.18608/jla.2021.7288

Hao, J., Liu, L., von Davier, A. A., Lederer, N., Zapata-Rivera, D., Jakl, P., \& Bakkenson, M. (2017). EPCAL: ETS platform for collaborative assessment and learning. ETS Research Report Series, 2017(1), 1-14.

https://doi.org/10.1002/ets2.12181

Hassan, J., Leong, J., \& Schneider, B. (2021). Multimodal data collection made easy: The EZ-MMLA Toolkit. 11th International Conference on Learning Analytics and Knowledge (LAK '21), 12-16 April 2021, Online, Everywhere.

Hudson, M. E., Voytecki, K. S., \& Zhang, G. (2018). Mixed-reality teaching experiences improve preservice special education students' perceptions of their ability to manage a classroom. Journal for Virtual Worlds Research, 11(2). https://doi.org/10.4101/jvwr.v11i2.7308

Janssen, J., Erkens, G., \& Kirschner, P. A. (2011). Group awareness tools: It's what you do with it that matters. Computers in Human Behavior, 27(3), 1046-1058. https://doi.org/10.1016/j.chb.2010.06.002

Kitto, K., Buckingham Shum, S., \& Gibson, A. (2018). Embracing imperfection in learning analytics. In Proceedings of the Eighth International Conference on Learning Analytics and Knowledge (LAK '18), 5-9 March 2018, Sydney, NSW, Australia (pp. 451-460). New York: ACM. https://doi.org/10.1145/3170358.3170413

Lämsä, J., Uribe, P., Jiménez, A., Caballero, D., Hämäläinen, R., \& Araya, R. (2021). Deep networks for collaboration analytics: Promoting automatic analysis of face-to-face interaction in the context of inquiry-based learning. Journal of Learning Analytics, 8(1), 113-125. http://doi.org/10.18608/jla.2021.7118

Martinez-Maldonado, R., Gašević, D., Echeverria, V., Fernandez Nieto, G, Swiecki, Z., \& Buckingham Shum, S. (2021). What do you mean by collaboration analytics? A conceptual model. Journal of Learning Analytics, 8(1), 126-153. http://doi.org/10.18608/jla.2021.7227

Markauskaite, L., Muukkonen, H., Damsa, C., Thompson, K., Arthars, N., Celik, I., ... Kali, Y. (2020). Interdisciplinary learning in undergraduate and graduate education: Conceptualizations and empirical accounts. In M. Gresalfi \& I. S. Horn (Eds.), The Interdisciplinarity of the Learning Sciences, 14th International Conference of the Learning Sciences (ICLS 2020), 19-23 June 2020, Nashville, TN, USA (Volume 1, pp. 398-405). International Society of the Learning Sciences. https://repository.isls.org/handle/1/6664

Martinez-Maldonado, R., Schulte, J., Echeverria, V., Gopalan, Y., \& Buckingham Shum, S. (2020). Where is the teacher? Digital analytics for classroom proxemics. Journal of Computer Assisted Learning, 36(5), 741-762.

https://doi.org/10.1111/jcal.12444

Metz, C. (2019, November 11). We teach A.I. systems everything, including our biases. The New York Times. https://www.nytimes.com/2019/11/11/technology/artificial-intelligence-bias.html

Ochoa, X., \& Worsley, M. (2016). Augmenting learning analytics with multimodal sensory data. Journal of Learning Analytics, 3(2), 213-219. https://doi.org/10.18608/jla.2016.32.10

O'Neil, C. (2016). Weapons of Math Destruction: How Big Data Increases Inequality and Threatens Democracy. Broadway Books.

Pennington, D., Bammer, G., Danielson, A., Gosselin, D., Gouvea, J., Habron, G., ... Wei, C. (2016) National The EMBeRS project: Employing model-based reasoning in socio-environmental synthesis. Journal of Environmental Studies and Sciences, 6, 278-286. https://doi.org/10.1007/s13412-015-0335-8

Pennington, D., Vincent, S., Gosselin, D., \& Thompson, K. (in press). Learning across Disciplines in Socio-Environmental Problem Framing. Socio-Environmental Systems Modeling.

Penuel, W. R., \& Gallagher, D. J. (2017). Creating Research Practice Partnerships in Education. Cambridge, MA, USA: Harvard Education Press.

Rai, A. (2020). Explainable AI: From black box to glass box. Journal of the Academy of Marketing Science, 48(1), 137-141. https://doi.org/10.1007/s11747-019-00710-5

Reimann, P. (2013). Design-based research: Designing as research. In R. Luckin, S. Puntambekar, P. Goodyear, B. Grabowski, J. Underwood, \& N. Winters (Eds.), Handbook of Design in Educational Technology (pp. 44-52). Routledge.

Salomon, G., \& Globerson, T. (1989). When teams do not function the way they ought to. International Journal of Educational Research, 13(1), 89-99. https://doi.org/10.1016/0883-0355(89)90018-9

Siemens, G. (2013). Learning analytics: The emergence of a discipline. American Behavioral Scientist, 57(10), 1380-1400. https://doi.org/10.1177/0002764213498851

Soller, A., Martínez, A., Jermann, P., \& Muehlenbrock, M. (2005). From mirroring to guiding: A review of state of the art technology for supporting collaborative learning. International Journal of Artificial Intelligence in Education, 15(4), 261-290. https://dl.acm.org/doi/10.5555/1434935.1434937 
Schwendimann, B. A., Rodriguez-Triana, M. J., Vozniuk, A., Prieto, L. P., Boroujeni, M. S., Holzer, A., ... Dillenbourg, P. (2016). Perceiving learning at a glance: A systematic literature review of learning dashboard research. IEEE Transactions on Learning Technologies, 10(1), 30-41. https://doi.org/10.1109/TLT.2016.2599522

Swiecki, Z. (2021). Measuring the impact of interdependence on individuals during collaborative problem-solving. Journal of Learning Analytics, 8(1), 75-94. http://doi.org/10.18608/jla.2021.7240

Webster, K., Costa-jussà, M. R., Hardmeier, C., \& Radford, W. (2019). Gendered ambiguous pronoun (GAP) shared task at the Gender Bias in NLP Workshop 2019. In Proceedings of the First Workshop on Gender Bias in Natural Language Processing, 1-2 August 2019, Florence, Italy (pp. 1-7). https://doi.org/10.18653/v1/W19-3801

Winne, P. H. (2017). Leveraging big data to help each learner and accelerate learning science. Teachers College Record, 119(3), 1-24. https://psycnet.apa.org/record/2017-15518-001

Wise, A. F., \& Shaffer, D. W. (2015). Why theory matters more than ever in the age of big data. Journal of Learning Analytics, 2(2), 5-13. https://doi.org/10.18608/jla.2015.22.2

Wise, A. F., \& Schwarz, B. B. (2017). Visions of CSCL: Eight provocations for the future of the field. International Journal of Computer-Supported Collaborative Learning, 12(4), 423-467. https://doi.org/10.1007/s11412-017-9267-5

Wise, A. F. (2019). Learning analytics: Using data-informed decision-making to improve teaching and learning. In O. O. Adesope \& A. G. Rud (Eds.), Contemporary Technologies in Education: Maximizing Student Engagement, Motivation, and Learning (pp. 119-143). Springer International. https://doi.org/10.1007/978-3-319-89680-9 7

Worsley, M., Anderson, K., Melo, N., \& Jang, J. Y. (2021). Designing analytics for collaboration literacy and student empowerment. Journal of Learning Analytics, 8(1), 30-48. http://doi.org/10.18608/jla.2021.7242 